\title{
Effect of Humidity, OH Radicals, UV Irradiation, Adhesive and Adsorption Effect on Photocatalyst Sterilization
}

\section{Shintani $H^{*}$}

Faculty of Science and Engineering, Chuo University, 1-13-27, Kasuga, Bunkyo, 112-8551, Tokyo, Japan

The photocatalyst equipment consists of a titanium dioxide $\left(\mathrm{TiO}_{2}\right)$ membrane and an ultraviolet (UV) lamp. The authors studied lf the photocatalyst equipment was practically useful in sterilizing environmental microorganisms in the health care facility. The number of microorganisms (CFU, colony forming unit) was compared in the cases of no sterilization (control), UV irradiation alone and photocatalyst sterilization. As a result, a statistical difference was observed between control and the photocatalyst sterilization against airborne microorganisms $(p<0.01)$ but not against surface microorganisms $(p>0.2)$. The photocatalyst uses an air sucking system, so it may be ineffective against microorganisms tightly attached to surfaces (adhesive microorganisms). However, the effectiveness and significant difference compared with control of the photocatalyst to sterilize airborne microorganisms in the health care facility was successfully confirmed.

Concerning the humidity effect on the photocatalyst sterilization, the authors compared the number of airborne microorganisms in cases of the control, UV alone and photocatalyst sterilization when humidity was changed. A statistical difference was obs erved between UV alone and the photocatalyst sterilization $(p<0.01)$ when humidity ( $\mathrm{RH}$, relative humidity) was increased to $60-70 \%$ (Figure 1), but not observed between UV alone and the photocatalyst sterilization ( $\mathrm{p}>0.2)$ when humidity was controlled to $10-20 \%$ (Figure 2). This indicates that maintaining relatively higher humidity levels will present satisfactory sterilization results due to a greater production of $\mathrm{OH}$ radicals. From Figure 2, when humidity level was low, $\mathrm{OH}$ radical effect was not significant because no difference between photocatalyst and UV. Surely they present lower level of airborne microorganisms compared control (Figures 1 and 2), but this is mainly due to UV effect, not $\mathrm{OH}$ radical effect because $\mathrm{OH}$ radical was effected by humidity compared with UV.

The UV irradiation sterilization was mainly surface sterilization and not always sufficiently effective against fungi, but effective against bacteria including spore formers. Fungi are most tolerant to UV sterilization than bacteria including spore formers. Fungi tolerate UV irradiation sterilization compared with bacteria including spore formers. The fungus most tolerant to UV irradiation steriliz ation was Aspergilus niger. The next tolerable fungus was Rhizopus spp.

UV or pulsed light irradiation alone may be sufficient to sterilize fungi. In that sense, the synergistic effect of $\mathrm{OH}$ radical and UV exposure when humidity was relatively rich at $\mathrm{RH} 60-70 \%$ (Figure 1) may be effective. This is the same as reported for the synergistic effect of UV irradiation and $\mathrm{H}_{2} \mathrm{O}_{2}$ gas exposure. The speculation synergistic effect of $\mathrm{UV}$ and $\mathrm{OH}$ radical contribution in the photocatalyst sterilization may be correct (Figure 1). However, the favorable synergistic effect of $\mathrm{OH}$ radical and UV irradiation was only when humidity was sufficient (Figure 1). When humidity was insufficient at RH 10-20\% (Figure 2), the synergistic effect was unsatisfactory and the effect on airborne microorganism by $\mathrm{UV}$ alone and $\mathrm{UV}+\mathrm{OH}$ radical was the same (Figure 2). This was due to the insufficient production of $\mathrm{OH}$ radicals from UV irradiated water. This was statistically confirmed from the comparison of UV $v s$. photocatalyst sterilization when humidity was sufficient (Figure 1) and insufficient (Figure 2). Maintaining the humidity at a higher degree is an essential factor to attain significant and reproducible photocatalyst efficiency to avoid any seasonable variation in performance.

From the comparison between Figures 1 and 2, sufficient humidity was indispensable to attain satisfactory sterilization effect against bacteria and fungi. UV sterilization efficiency was reported to decrease when the humidity is high by Ko et al. but the increase in $\mathrm{OH}$ radicals by increasing humidity could overcome the reported diminishment of UV irradiation efficiency and a desirable sterilization result was obtained. This indicates that the interference of humidity in UV sterilization was not significant contrary to the results reported by Ko et al. [1].

To conduct the statistical analysis of data in Figures 1 and 2, data were transformed to the square root value to perform the Student $t$ test, ANOVA (analysis of variation) and/or $\chi^{2}$ test because the distribution of the environmental microorganisms is the Poisson distribution not the normal distribution. The $p$ value determined by the paired Student $t$ test for the airborne microorganisms using the

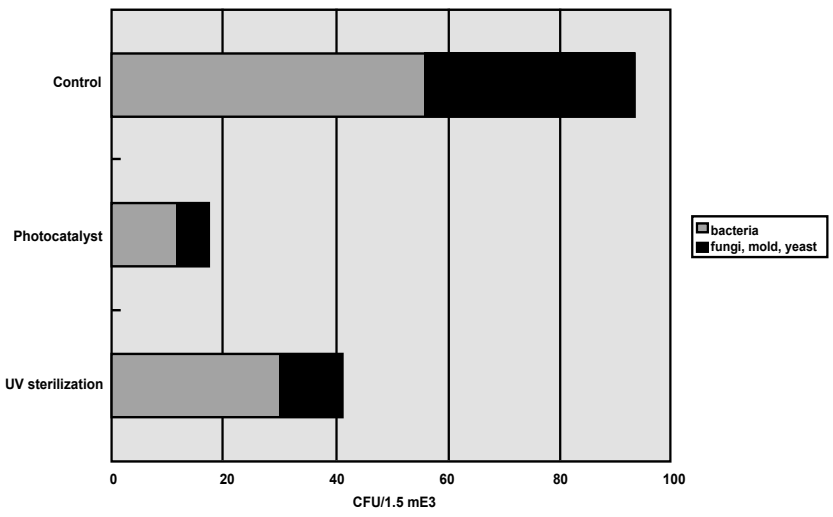

Figure 1: Results from the control, photocatalyst and UV sterilization of airborne bacteria and fungi when humidity was controlled to $60-70 \%$.

*Corresponding author: Shintani $\mathrm{H}$, Faculty of Science and Engineering, Chuo University, 1-13-27, Kasuga, Bunkyo, 112-8551, Tokyo, Japan, Tel: +81425922336; Fax +81425922336; E-mail: shintani@mail.hinocatv.ne.jp

Received September 22, 2014; Accepted September 24, 2014; Published September 30, 2014

Citation: Shintani H (2014) Effect of Humidity, OH Radicals, UV Irradiation Adhesive and Adsorption Effect on Photocatalyst Sterilization. Chem Sci J 5: e104. doi: $10.4172 / 2150-3494.1000$ e104

Copyright: (c) 2014 Shintani H. This is an open-access article distributed under the terms of the Creative Commons Attribution License, which permits unrestricted use, distribution, and reproduction in any medium, provided the original author and source are credited. 


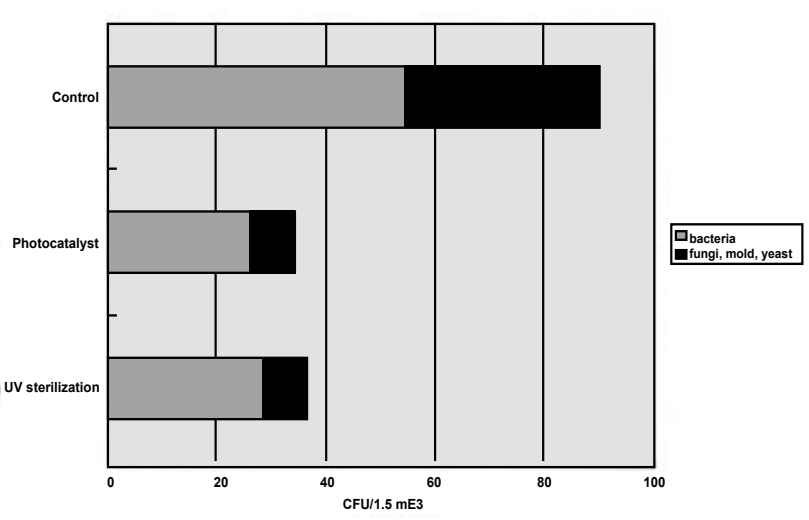

Figure 2: Results from the control, photocatalyst and UV sterilization of airborne bacteria and fungi when humidity was controlled to $10-20 \%$.

square root value was conducted. The Student $t$ test result from the data transformed to the square root values and that of ANOVA result was identical. The result of $\chi^{2}$ test was somewhat different from that of Student $t$ test and that of ANOVA, however a tendency was identical. A statistically significant difference could be observed between the airborne microorganisms subject to the control and photocata lyst treatments $(p<0.01$, Figure 1$)$. On the contrary, no statistical difference $(p>0.2)$ could be observed between photocatalyst and UV sterilization in Figure 2 for both bacteria and fungi when the humidity was controlled at lower lever of 10-20 RH \%. However, a statistical difference $(p<0.01)$ in bacteria and fungi could be observed between those receiving photocatalyst and UV exposure in Figure 1 when humidity was maintained at $60-70 \%$. The photocatal yst sterilization performed well even though the UV function is thought to be somewhat decreased at high humidity as reported by Ko et al.. However, it is speculated that humidity at more than $70 \%$ would show inferior results to those obtained at $60-70 \%$ due to insufficiency of UV function as reported by Ko et al. [1].

In addition, it may be obvious that no significant differen ce in the CFU from air samples could be observed between the control and the photocatalyst equipment turned off UV irradiation. This indicates the adsorption phenomena of environmental microorganisms onto the $\mathrm{TiO}_{2}$ membrane with ion exchange, ionic bonding, hydrogen bonding or Van der Waals bonding was not significant. This can also be explained from the comparison between photocatalyst and UV irradiation in Figure 2 compared with that in Figure 1. The reason for the decrease in $\mathrm{C}$
FU by photocatalyst treatment in Figure 1 was not due to increasing adsorption but increasing $0 \mathrm{H}$ radicals by increasing humidity at 60 $70 \mathrm{RH} \%$, which results in satisfactory sterilization. In addition, if adsorption phenomenon was significant, it could not be satisfactory explained no significant difference between UV alone and photocatalyst in Figure 2.

Even though $\mathrm{OH}$ radicals cannot be directly determined with ESR or chemiluminescence as $\mathrm{OH}$ radicals exist in the interior of the photocatalyst equipment, we can speculate that the $\mathrm{OH}$ radicals will increase within the photocatalyst with

increasing humidity up to $\mathrm{RH} 60-70 \%$. This is because we determined stable radicals after photocatalyst sterilization and obtained the result that the stable radicals increased at higher humidity by ESR compared with fewer stable radicals at lower humidity levels. It is necessary to note that the life period of active radicals is quite short $(\mathrm{OH}$ radical is $\mu \mathrm{s})$ and the produced amount of the active $\mathrm{OH}$ radicals is not abundant. In addition the production of stable radicals by increasing the humidity may not always parallel the produced amount of active $\mathrm{OH}$ radicals. It may be sure that active $\mathrm{OH}$ radicals will increase with increasing humidity up to 60 to $70 \%$. In addition, there was no data supporting the adsorption phenomenon of environmental microorganisms on any membranes used in the photocatalyst sterilization, thus the adsorption phenomenon on to membrane can be denied in the photocatalyst function.

From the result, it was clarified that sufficient humidity for the photocatalyst equipment improves the sterilization efficiency. Humidity is an indispensable factor to increase the efficiency of the photocatalyst function.

The single exposure period of airborne microorganisms to $\mathrm{OH}$ radicals within the photocatalyst is quite short period. It must be lengthened to attain greater sterilization efficiency. In addition, $\mathrm{OH}$ radicals from the irradiated $\mathrm{TiO}_{2}$ must be increased to improve the sterilization effect of the photocatalyst on airborne microorganisms.

By lengthening the exposure period within the chamber and increasing the $\mathrm{OH}$ radical formation, photocatalyst sterilization efficiency may be much improved and as a result nosocomial infectious disease in the health care facility may be decreased in future when the improved photocatalyst equipment becomes available.

\section{References}

1. Ko G, First MW, Burge HA (2000) Influence of relative humidity on particle size and UV sensitivity of Serratia marcescens and Mycobacterium bovis BCG aerosois. Tuber Lung Dis 80: 217-228. 